Case Report

\title{
A Rare Case of Ovarian Serous Borderline Tumor with Brain Metastasis
}

\author{
Sonia Veran-Taguibao (iD, Roberto Alvaro A. Taguibao, Nicolas Gallegos (D), \\ Ted Farzaneh, Ronald Kim, Fritz Lin, and Di Lu 1 \\ University of California, Irvine Health System, Department of Pathology, Division of Anatomic Pathology, Orange, CA, USA \\ Correspondence should be addressed to Di Lu; lud5@uci.edu
}

Received 17 October 2018; Accepted 13 April 2019; Published 22 May 2019

Academic Editor: Alp Usubutun

Copyright (C) 2019 Sonia Veran-Taguibao et al. This is an open access article distributed under the Creative Commons Attribution License, which permits unrestricted use, distribution, and reproduction in any medium, provided the original work is properly cited.

Background. Serous borderline tumor represents a group of noninvasive tumor of the ovary bridging between benign serous cystadenoma and serous carcinoma. They are commonly seen in younger women and usually have an excellent outcome but seldom show local recurrence (J. F. Leake et al. 1991). Metastasis to the lymph nodes has rarely been reported (M. D. Chamberlin et al., 2001; M. B. Verbruggen et al., 2006). Moreover, the brain is exceptionally a rare metastatic site for ovarian tumor. There is one case of an advanced staged SBT with micropapillary pattern metastasis to the brain recently and by far it is the most distant metastasis reported (M. D. Martin et al., 2017). However, to the best of our knowledge, no report has been documented for a recurrent stage 1 typical SBT metastasizing to the brain.

\section{Introduction}

Approximately 3000 American women are diagnosed with borderline ovarian tumors (BOTs) annually and account for $15-20 \%$ of all ovarian epithelial tumors. They are known as a tumor of low malignant potential (LMP) or as an atypical proliferative tumor. They are characterized by an increased epithelial proliferation accompanied by nuclear atypia (usually mild to moderate) and low mitotic index in the absence of infiltrative destructive growth or obvious stromal invasion. Histologically, they are subdivided into serous (53.3\%), mucinous $(42.5 \%)$, and the less common, mixed, endometrioid, clear cell, or Brenner tumor (4.2\%) [1]. This current case report focuses on serous borderline tumors (SBTs).

SBTs account for one-fourth to one-third of the serous tumors $[1,2]$. They occur commonly in the fourth and fifth decades, with an average patient age of 42 years. Although often asymptomatic, the tumor may sometimes present with abdominal enlargement and pain due to rupture or torsion. Approximately $70 \%$ of this tumor is confined to one or both ovaries (stage I) at the time of diagnosis. The remaining tumors are found within the pelvis (stage II) or upper abdomen (stage III) and only rare cases have extended beyond the abdomen (stage IV) at the time of presentation [3].
Serous borderline tumor is further subclassified into typical serous borderline tumor, also known as atypical proliferative serous tumor (APST), and the micropapillary variant of serous borderline tumor, which is variably referred to as noninvasive micropapillary serous carcinoma. Typical serous borderline tumor represents approximately three-fourths of the SBTs and is associated with a favorable prognosis, while micropapillary SBT is approximately one-third of the SBT and is associated with unfavorable prognosis.

They are known to spread transperitoneally. Few authors described the spread of this tumor to regional lymph nodes, supradiaphragmatic lymph nodes [4], and internal mammary lymph nodes [5].

It is exceedingly rare to spread to the brain owing to more effective treatment of the primary cancer. However, if it does occur, the most common epithelial ovarian carcinoma associated with this incidence is of serous histotype $[6,7]$.

\section{Case Report}

We present a 33-year-old nulliparous female who presented at our institution with a 3-year progressive headache and was associated with expressive aphasia. MRI of the brain revealed 4 masses including 2 dominant mass lesions $(6.0$ and $4.5 \mathrm{~cm})$ 


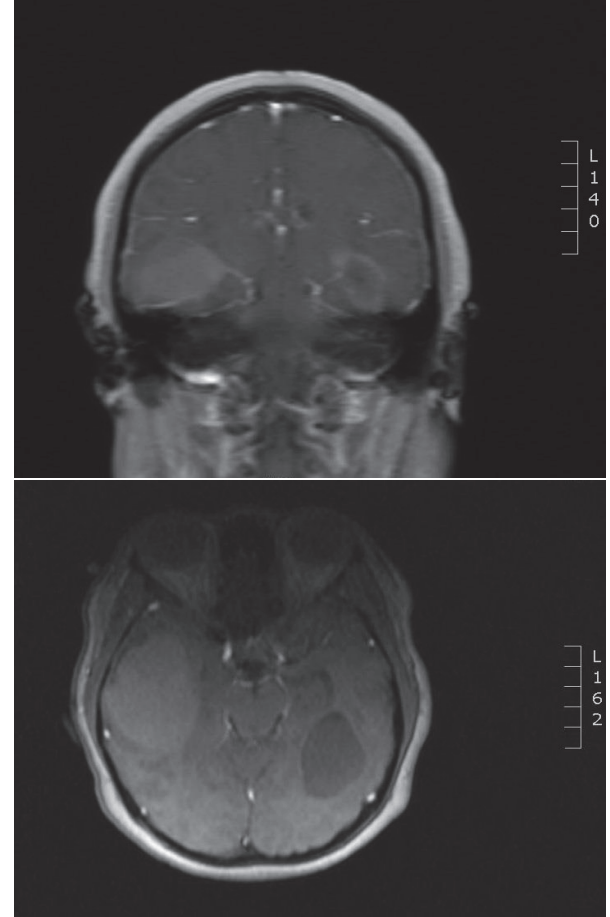

FIGURE 1: MRI of the brain was done which revealed 4 masses with 2 dominant mass lesions $(6.0$ and $4.5 \mathrm{~cm}$ ) having irregular lobulations in the bilateral temporal lobes consistent with metastatic disease.

having irregular lobulations in the bilateral temporal lobes consistent with metastatic disease (Figure 1). Past medical history revealed that unilateral salpingo-oophorectomy with omentectomy, peritoneal washing, and pelvic lymph node samplings were performed twice, 8 and 4 years prior, respectively. Both specimens had serous borderline tumor, one of which had a $1 \mathrm{~mm}$ focus of microinvasion.

The fluid sample from the current cystic mass in the brain revealed neoplastic cells forming papillary clusters with smooth contoured edges on the smear (Figure 2(a)). Tissue sample of the brain lesion showed clusters of broad papillae with hierarchical branching and is lined by polygonal to columnar serous epithelium with mild to moderate atypia (Figure 2(b)). Immunohistochemical staining shows positive staining for PAX 8, WT-1, and CK7 and negative staining for CK20 (Figures 2(c)-2(f)). The morphologic features and immunoprofile are in keeping with a diagnosis of the previous ovarian tumor.

\section{Discussion}

The risk factors for SBTs are similar to those for ovarian cancer with notable exceptions of a higher frequency of infertility and a lower frequency of BRCA mutations [8]. The risk factors for recurrent SBTs includes FIGO stage, presence of implants, micropapillary pattern, and microinvasion. Additional factors suggested are incomplete surgical staging, residual disease, fertility-sparing surgery, bilateral ovarian involvement, capsular rupture, and age $[9,10]$.
Reproductive age group with Stage I SBT, with or without noninvasive implants, can be treated conservatively. According to recent data, recurrence is infrequent ranging from 1.8 to $15 \%$. Therefore, conservative surgery with careful surgical exploration is sufficient [11-13]. In addition, recurrence or development of a new SBT can be effectively treated with reoperation alone for these patients.

Recurrence, though not commonly observed, can occur in a residual ovary especially following fertility-sparing surgery $[1,14]$. Preserving the uterus and ovary increases the risk of disease recurrence in the remaining ovary due to the possibility of bilateral synchronous tumors or occult metastases left in situ especially in serous histotype.

Silva et al. observed that, in the recurrences of 11 (6.8\%) out of 160 Stage I SBTs treated with total abdominal hysterectomy with bilateral salpingo-oophorectomy, cases showed a higher frequency of endosalpingiosis (72.7\%). Thus they suggest that late recurrent tumors could represent new primary serous tumor arising from endosalpingiosis [15]. Another study suggested that glandular inclusions (müllerian cysts) resembling endosalpingiosis seen in the lymph node sample could be a bland appearing form of metastatic SBTs $[8,16]$.

Although controversial, microinvasion was said to be linked to the recurrence of SBTs. Review of the literatures showed that the overall differences were not statistically significant between typical SBT and SBT with stromal invasion with or without micropapillary features [11]. A retrospective study by Ferrero A et al. compared 209 patients with borderline ovarian tumors (BOT). The microinvasive BOTs had higher recurrence rates $(21 \%)$ when compared to BOTs without microinvasion (12\%), with a median follow-up of 53 months. However, the report also did not yield a statistical significance and the staging for the serous histologic type was not well documented. Seidman JD et al. revealed that stromal microinvasion, if unassociated with extraovarian invasive implants, has no effect on the rate of recurrence or the rate of progression to invasive disease as confirmed in a large metaanalysis [11, 13]. Moreover, overall survival rate for patient with stromal microinvasion that have had stage I disease is at most $91 \%$. Therefore, there is no suggestion for change in the current management [17].

Invasive and noninvasive implants are seen in $35 \%$ of the typical SBT patients. However, the invasive implants are known to have higher relapse rate ( $>50 \%)$. Invasive implants are also strongly associated with micropapillary architecture and have worse prognosis than noninvasive implants $[1,9]$. If an invasive implant is found in a typical SBT, it suggests insufficient sampling [1] with possible unsampled micropapillary areas or areas of microinvasion. Thus, the current guideline recommends tissue submission of 2 sections per $1 \mathrm{~cm}$ for serous histotype tumors for better evaluation of the tumor morphologically [18]. Nonetheless, complete surgical staging and restaging surgery, though controversial, are endorsed for the detection of extra ovarian peritoneal implants which may better assist in the prognosis [1, 19-21].

Other factors associated with recurrence are capsular rupture owing a hazard ratio of 1.9; 95\% CI: 1.1-3.6 for recurrent APST and HR of 1.7; 95\% CI: 0.4-7.5 for subsequent 


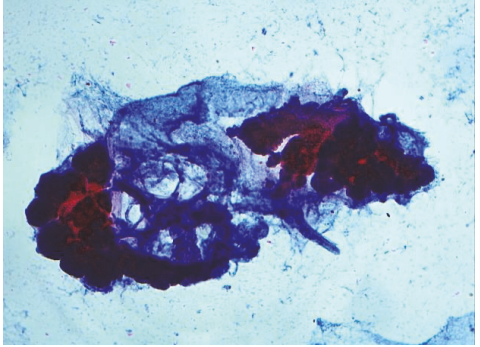

(a)

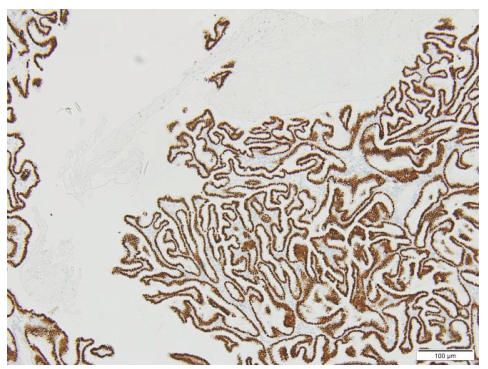

(d)

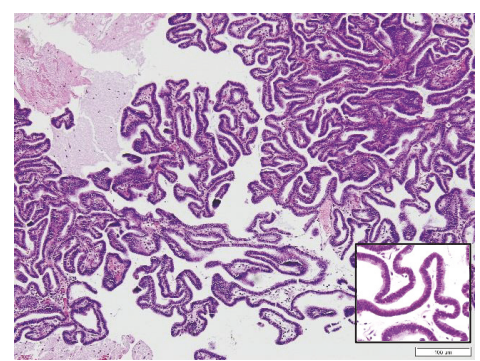

(b)

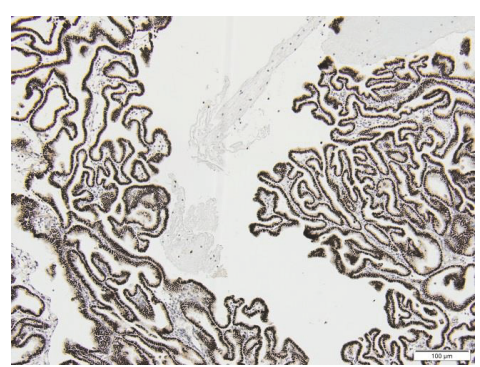

(e)

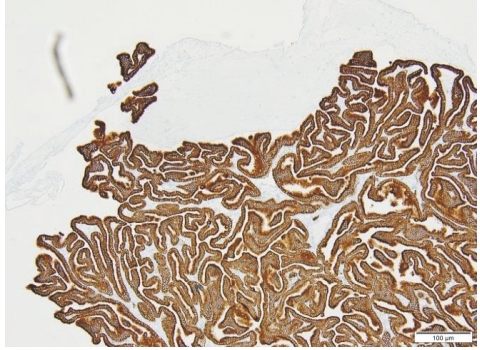

(c)

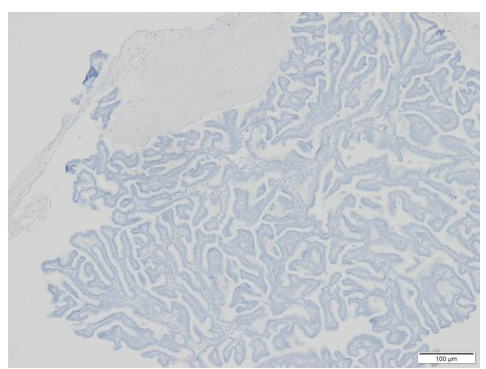

(f)

FIGURE 2: (a) Neoplastic cells forming papillary clusters with smooth contoured edges (b) Serous borderline tumor with hierarchically branched papillae lined by polygonal to columnar serous epithelium with mild atypia (inset) Immunohistochemistry showed (c) CK7 cytoplasmic positivity (d) PAX8 diffuse nuclear positivity (e) WT-1 diffuse nuclear positivity (f) CK20 negative staining.

serous carcinoma [10], and genetic mutations of KRAS and BRAF $[22,23]$.

Our patient who had previous procedures was clinically stage as FIGO stage I. However, the current procedure in our institution disclosed brain metastasis. A review of the outside pathology reports, except for the $1 \mathrm{~mm}$ focus of microinvasion, did not show implants or micropapillary pattern, nor capsular rupture. The age of our patient suggested a very early onset ovarian tumor, but next generation sequencing panel for ATM, BARD1, BRCA1, BRCA2, BRIP1, CDH1, CHEK2, EPCAM, MRE11A, MLH1, MSH2, MSH6, MUTYH, NBN, NF1, PALB2, PMS2, PTEN, RAD50, RAD51C, RAD51D, SMARCA4, STK11, and TP53 did not reveal pathogenic mutations, gross deletions, and duplications. No KRAS and BRAF study was done in our patient. She has been treated with brain tumor resection and fractions of intensity-modulated radiotherapy (IMRT) without complications to the resection cavities.

She is followed-up in every 4-month interval and so far, her brain MRI showed no evidence of disease progression. Twenty-three months after her last procedure, she is stable and shows no signs of deterioration.

\section{Conclusion}

To our knowledge, this report represents a rare case of serous borderline tumor metastasizing to the brain. To create a coordinated plan of care for patients with serous borderline tumor, the clinical, surgical, and pathological management of ovarian tumors is of outmost importance in accurate diagnosis and staging of the tumor.

\section{Disclosure}

The abstract of this manuscript has been presented in the ASCP Annual Meeting 2017. The authors have not received financial or funding in the preparation of this report.

\section{Conflicts of Interest}

The authors declared no conflicts of interest.

\section{Authors' Contributions}

Dr. Ted Farzaneh contributed in the abstract presented during the ASCP Annual Meeting 2017.

\section{References}

[1] D. Fischerova, M. Zikan, P. Dundr, and D. Cibula, "Diagnosis, treatment and follow-up of borderline ovarian tumors," The Oncologist, vol. 17, no. 12, pp. 1515-1533, 2012.

[2] R. Hogg, J. Scurry, S.-N. Kim, M. Friedlander, and N. Hacker, "Microinvasion links ovarian serous borderline tumor and grade 1 invasive carcinoma," Gynecologic Oncology, vol. 106, no. 1, pp. 44-51, 2007.

[3] S. J. Robboy, G. L. Mutter, J. Prat, R. C. Bentley, P. Russell, and M. C. Anderson, Pathology of the Female Reproductive Tract, 2nd edition.

[4] J. F. Leake, J. S. Rader, J. D. Woodruff, and N. B. Rosenshein, "Retroperitoneal lymphatic involvement with epithelial ovarian tumors of low malignant potential," Gynecologic Oncology, vol. 42, no. 2, pp. 124-130, 1991. 
[5] M. D. Chamberlin, G. H. Eltabbakh, S. L. Mount, and B. J. Leavitt, "Metastatic serous borderline ovarian tumor in an internal mammary lymph node," Gynecologic Oncology, vol. 82, no. 1, pp. 212-215, 2001.

[6] Z. R. Cohen, D. Suki, J. S. Weinberg et al., "Brain metastases in patients with ovarian carcinoma: prognostic factors and outcome," Journal of Neuro-Oncology, vol. 66, no. 3, pp. 313-325, 2004.

[7] K. Pietzner, G. Oskay-Oezcelik, K. El Khalfaoui, D. Boehmer, W. Lichtenegger, and J. Sehouli, "Brain metastases from epithelial ovarian cancer: Overview and optimal management," Anticancer Reseach, vol. 29, no. 7, pp. 2793-2798, 2009.

[8] R. J. Kurman, L. H. Ellenson, and B. M. Ronnett, Blaustein's Pathology of Female Genital Tract, New York, NY, USA, 6th edition edition, 2011.

[9] D. M. Gershenson, "Clinical management potential tumours of low malignancy," Best Practice \& Research Clinical Obstetrics \& Gynaecology, vol. 16, no. 4, pp. 513-527, 2002.

[10] C. G. Hannibal, R. Vang, J. Junge, K. Frederiksen, R. J. Kurman, and S. K. Kjaer, "A nationwide study of ovarian serous borderline tumors in Denmark 1978-2002. Risk of recurrence, and development of ovarian serous carcinoma," Gynecologic Oncology, vol. 144, no. 1, pp. 174-180, 2017.

[11] J. Prat and M. De Nictolis, "Serous borderline tumours of the ovary. A long term follow up of 137 cases, including 18 with a micropapillary pattern and 20 with microinvasion," The American Journal of Surgical Pathology, vol. 26, no. 9, pp. 11111128, 2002.

[12] A. W. Kennedy and W. R. Hart, "Ovarian papillary serous tumors of low malignant potential (serous borderline tumors): a long term follow up study, including patients with microinvasion, lymph node metastasis, and transformation to invasive serous carcinoma," Cancer, vol. 78, no. 2, pp. 278-286, 1996.

[13] J. D. Seidman and R. J. Kurman, "Ovarian serous borderline tumors; a critical review of the literature with emphasis on prognostic indicators," Human Pathology, vol. 31, no. 5, pp. 539$557,2000$.

[14] A. Ferrero, I. Strada, B. Di Marcoberardino et al., "Clinical significance of microinvasion in borderline ovarian tumors and its impact on surgical management," International Journal of Gynecologic Cancer, vol. 22, no. 7, pp. 1158-1162, 2012.

[15] E. G. Silva, C. Tornos, Z. Zhuang, M. I. Merino, and D. M. Gershenson, "Tumor recurrence in stage I ovarian serous neoplasms of low malignant potential," International Journal of Gynecological Pathology, vol. 17, pp. 1-6, 1998.

[16] W. F. Moore, R. C. Bentley, A. Berchuck, and S. J. Robboy, "Some mullerian inclusion cysts in lymph nodes may sometimes be metastases from serous borderline tumor of the ovary," The American Journal of Surgical Pathology, vol. 25, pp. 710-718, 2000.

[17] J. K. McKenney, B. L. Balzer, and T. A. Longacre, "Patterns of stromal invasion in ovarian serous tumors of low malignant potential (borderline tumors): a reevaluation of the concept of stromal microinvasion," The American Journal of Surgical Pathology, vol. 30, no. 10, pp. 1209-1221, 2006.

[18] J. D. Seidman, F. Khedmati, and A. V. Yemelyanova, "Ovarian low grade serous neoplasm: evaluation of sampling recommendations based on tumors expected to have invasion (those with peritoneal invasive LGSC (invasive implants))," Modern Pathology, vol. 22, supplement 1, 2009.

[19] R. Fauvet, J. Boccara, C. Dufournet, E. David-Montefiore, C. Poncelet, and E. Daraï, "Restaging surgery for women with borderline ovarian tumors: results of a french multicenter study," Cancer, vol. 100, no. 6, pp. 1145-1151, 2004.

[20] I. Zapardiel, P. Rosenberg, M. Peiretti et al., "The role of restaging borderline ovarian tumors: Single institution experience and review of the literature," Gynecologic Oncology, vol. 119, no. 2, pp. 274-277, 2010.

[21] W. Winter III, P. R. Kucera, W. Rodgers et al., "Surgical staging in patients with ovarian tumors of low malignant potential," Obstetrics \& Gynecology, vol. 100, no. 4, pp. 671-676, 2002.

[22] C.-L. Ho, R. J. Kurman, R. Dehari, T.-L. Wang, and I.-M. Shih, "Mutations of BRAF and KRAS precede the development of ovarian serous borderline tumors," Cancer Research, vol. 64, no. 19, pp. 6915-6918, 2004.

[23] Y. T. Tsang, M. T. Deavers, C. C. Sun et al., "KRAS (but not BRAF) mutations in ovarian serous borderline tumor are associated with recurrent low grade serous carcinoma," The Journal of Pathology, vol. 231, no. 4, pp. 449-456, 2013. 


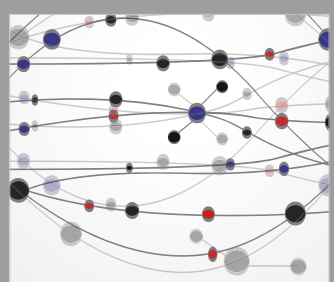

The Scientific World Journal
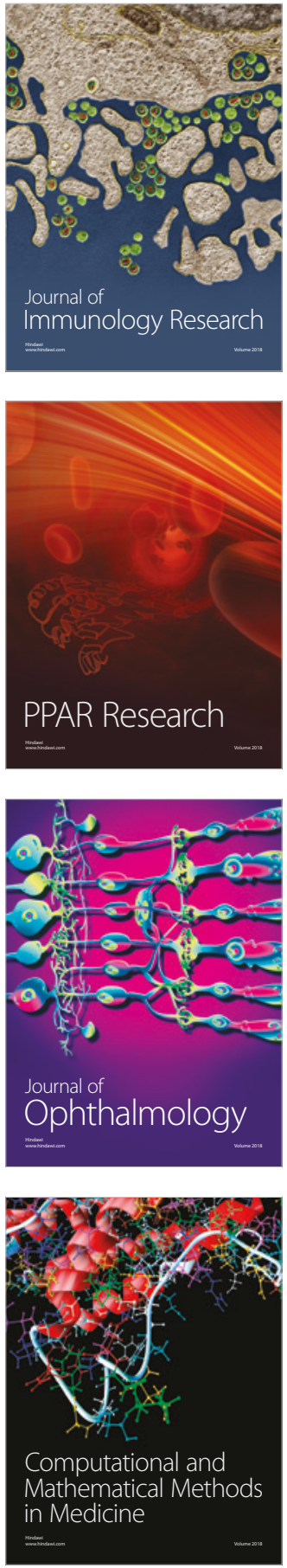

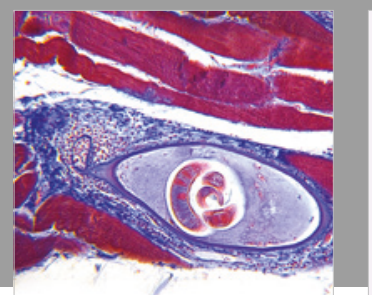

Gastroenterology Research and Practice

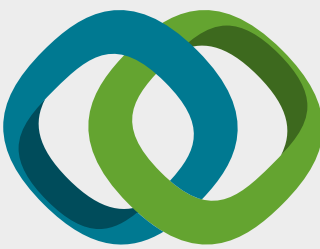

\section{Hindawi}

Submit your manuscripts at

www.hindawi.com
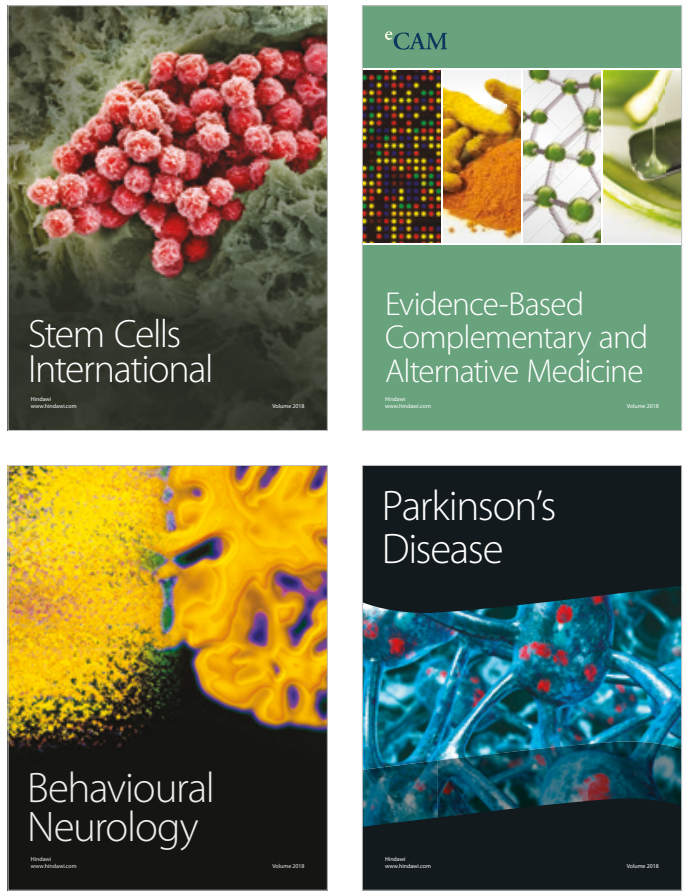

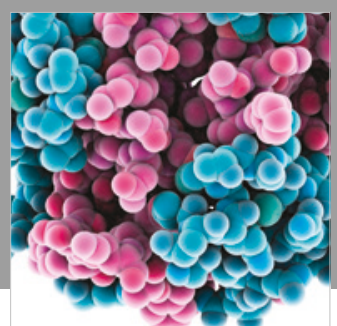

ournal of

Diabetes Research

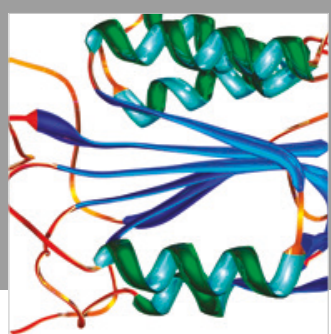

Disease Markers
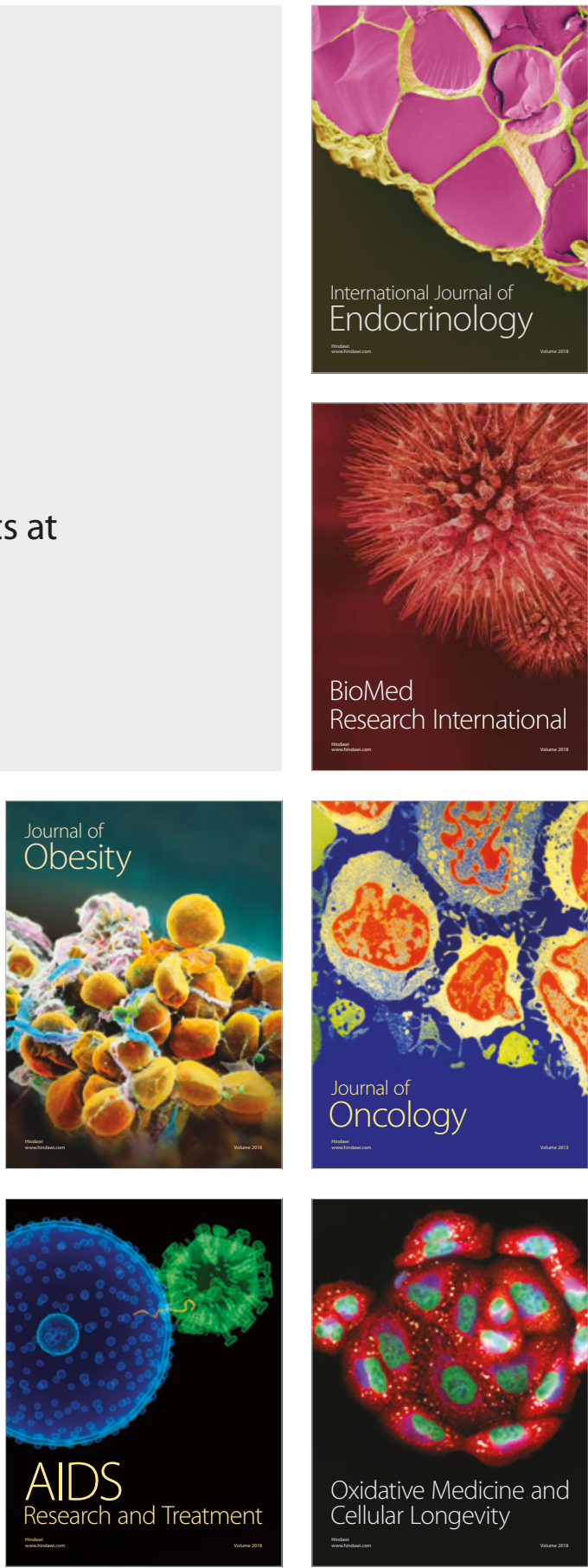